\title{
The effectiveness of digital interventions for increasing physical activity in individuals of low socioeconomic status: a systematic review and meta-analysis
}

\author{
Max J. Western ${ }^{1}$, Miranda E. G. Armstrong ${ }^{2}$, Ishrat Islam ${ }^{3}$, Kelly Morgan ${ }^{4}$, Una F. Jones ${ }^{5}$ and Mark J. Kelson ${ }^{6 *}$
}

\begin{abstract}
Background: Digital technologies such as wearables, websites and mobile applications are increasingly used in interventions targeting physical activity (PA). Increasing access to such technologies makes an attractive prospect for helping individuals of low socioeconomic status (SES) in becoming more active and healthier. However, little is known about their effectiveness in such populations. The aim of this systematic review was to explore whether digital interventions were effective in promoting PA in low SES populations, whether interventions are of equal benefit to higher SES individuals and whether the number or type of behaviour change techniques (BCTs) used in digital PA interventions was associated with intervention effects.

Methods: A systematic search strategy was used to identify eligible studies from MEDLINE, Embase, PsycINFO, Web of Science, Scopus and The Cochrane Library, published between January 1990 and March 2020. Randomised controlled trials, using digital technology as the primary intervention tool, and a control group that did not receive any digital technology-based intervention were included, provided they had a measure of PA as an outcome. Lastly, studies that did not have any measure of SES were excluded from the review. Risk of Bias was assessed using the Cochrane Risk of Bias tool version 2.
\end{abstract}

Results: Of the 14,589 records initially identified, 19 studies were included in the final meta-analysis. Using randomeffects models, in low SES there was a standardised mean difference (SMD $(95 \% \mathrm{Cl}))$ in PA between intervention and control groups of $0.06(-0.08,0.20)$. In high SES the SMD was $0.34(0.22,0.45)$. Heterogeneity was modest in both low $\left(I^{2}=0.18\right)$ and high $\left(I^{2}=0\right)$ SES groups. The studies used a range of digital technologies and BCTs in their interventions, but the main findings were consistent across all of the sub-group analyses (digital interventions with a PA only focus, country, chronic disease, and duration of intervention) and there was no association with the number or type of BCTs.

Discussion: Digital interventions targeting PA do not show equivalent efficacy for people of low and high SES. For people of low SES, there is no evidence that digital PA interventions are effective, irrespective of the behaviour change techniques used. In contrast, the same interventions in high SES participants do indicate effectiveness. To reduce inequalities and improve effectiveness, future development of digital interventions aimed at improving PA must make more effort to meet the needs of low SES people within the target population.

\footnotetext{
${ }^{*}$ Correspondence: M.J.Kelson@exeter.ac.uk

${ }^{6}$ Department of Mathematics/Institute of Data Science and Artificial Intelligence, University of Exeter, Laver Building, Exeter EX4 4QE, UK

Full list of author information is available at the end of the article
} permits use, sharing, adaptation, distribution and reproduction in any medium or format, as long as you give appropriate credit to the original author(s) and the source, provide a link to the Creative Commons licence, and indicate if changes were made. The images or other third party material in this article are included in the article's Creative Commons licence, unless indicated otherwise in a credit line to the material. If material is not included in the article's Creative Commons licence and your intended use is not permitted by statutory regulation or exceeds the permitted use, you will need to obtain permission directly from the copyright holder. To view a copy of this licence, visit http://creativecommons.org/licenses/by/4.0/. The Creative Commons Public Domain Dedication waiver (http://creativeco mmons.org/publicdomain/zero/1.0/) applies to the data made available in this article, unless otherwise stated in a credit line to the data. 
Keywords: Physical activity, Health inequalities, Behaviour change, RCT, Digital health, eHealth, Digital intervention, Socioeconomic status

\section{Background}

Physical activity (PA) incurs a multitude of health benefits and is consequently a cost-effective public health strategy for reducing the burden of non-communicable diseases [1]. The World Health Organisation reports that increasing physical activity levels worldwide could prevent 5 million premature deaths per year [2]. Exceeding the minimum recommended levels of PA can reduce the risk of colon and breast cancers, heart disease, stroke and diabetes by $20-30 \%[3,4]$. Recent estimates suggest that physical inactivity costs INT $\$ 54$ billion to health care systems across the world, of which around $80 \%$ is incurred by high-income countries [5]. Despite a surge in promotion efforts over the past two decades the prevalence of physical inactivity increased between 2001 and 2016 from 32 to $37 \%$ in high-income countries and remained twice as high as that in low-income countries [6].

Socioeconomic status (SES) is a term used to describe an individual's affluence or social standing, referencing factors such as wealth, educational level and occupation [7]. Many observers have found SES to associate with disparities in health and health behaviours, both withinand between-countries [8]. Globally, the life expectancy of a country's population can range from 52 years in the poorest countries to 84 years in the richest [9]. The main drivers of this inequality are thought to be discrepancies in education, income, and access to medicine, care and health information [10]. The same factors also predict life expectancy within a given country, with some of the richest countries demonstrating considerable discrepancies in terms of morbidity and mortality rates between high and low SES groups [11]. This pattern is also true for PA behaviour; in a recent UK survey, around $50 \%$ of adults in the most deprived quintile met the PA recommendations compared to $68 \%$ in the least deprived quintile [12]. Indeed, around the world, SES is thought to have a strong positive relationship with leisure-time PA [13-16], which is considered the PA domain best associated with overall health benefits $[17,18]$.

The rapid growth in number and sophistication of digital technologies such as websites, mobile or wearable devices, smartphone applications and telehealth or telemedicine have been presented as a cost-effective platform for promoting PA behaviour change and health improvement [19-21]. Access to such technologies are increasing around the globe, with internet penetration as high as $95 \%$ in the most developed nations and $60 \%$ worldwide [22]. Indeed, in the USA and UK over $90 \%$ of all adults own a smart phone, rising to around 95 and 99\% in 35-55- and 16-34-year old's respectively [23] implying that in these countries a large majority of the population across SES currently use such technologies. Digital technologies enable researchers and clinicians to develop remote interventions that are grounded in behavioural theory [24], can include a number of potentially useful behaviour change techniques [25] and can be tailored to meet the particular needs of a given individual or population [26]. Accordingly, digital technologies have been championed as a vehicle that reduces health inequalities by taking bespoke, informative and empowering programmes to otherwise hard to reach, low SES, populations [27].

Others, however, have argued that the use of digital technologies for health [behaviour] promotion can in fact create a 'digital divide' and that wearables and smartphone or web applications are predominantly designed for more affluent (and higher SES) people with higher levels of education and income [28, 29]. In particular, one's eHealth literacy (the term used to describe an individual's ability to seek out, comprehend, critique and act upon health-related knowledge and guidance delivered through digital means) is an important factor that can determine whether or not simply having the access to digital technologies promoting health behaviour change is actually useful to an individual [30-32]. A number of studies have demonstrated that low SES individuals tend to also have lower eHealth literacy and consequently do not incur the same benefits as their higher SES counterparts when engaging with digital health technologies [33-35].

Little is currently known about whether interventions deploying digital technologies to increase PA are equally effective in high and low SES populations. Systematic reviews indicate that the general effectiveness of eHealth interventions on PA behaviour change is modest yet promising [36-40]. However, neither the individual randomised controlled trials nor the pooled analysis included in these systematic-reviews have analysed their data in a way that separates the effects observed in higher and lower SES groups. This is important, as even digital behaviour change interventions that demonstrate a net overall effect on PA behaviour when comparing the intervention and control groups, may risk silently exacerbating health inequalities if its programme is effective for high SES populations but makes no difference to low SES populations. Moreover, analysing study populations 
as a whole does not inform us if the behaviour change techniques used in digital interventions are ubiquitously useful across SES groups. Behaviour change techniques (BCTs) refer to the active ingredients of a given intervention that aim to evoke a change in behaviour, which have been classified according to their nature such as goal setting, feedback and monitoring, and shaping knowledge [41]. Systematic reviews of PA interventions in other contexts have shown that the number of BCTs, which may be a marker of intervention complexity, does not necessarily dictate how effective an intervention may be $[42,43]$ but have not analysed their data according to SES. Understanding whether interventions have varying effectiveness for individuals of low SES compared to higher SES, and if any number or type of particular BCTs is particularly useful for low SES populations would help researchers and policymakers appropriately tailor their efforts towards reducing inequalities in PA promotion.

Accordingly, the aim of this systematic review and meta-analysis is to understand whether digital behaviour change interventions targeting increased PA are beneficial for low SES populations. Specifically, we set out to investigate the following research questions:

1. Are digital behaviour change interventions effective at promoting a change in PA behaviour when comparing the intervention with control groups amongst low SES participants?

2. Do digital behaviour change interventions promoting PA have equivalent effectiveness when we compare the effects of the intervention versus control group in high SES participants to the effects found in low SES participants [identified in research question 1]?

3 . Is the number or type of behaviour change techniques (BCTs) included in digital behaviour change interventions promoting PA associated with the study outcome in low and high SES groups?

\section{Methods}

The protocol for this systematic review is registered with the international prospective register for systematic reviews (PROSPERO, registration ID: CRD42018079540). The design and implementation of this review conform to the Preferred Reporting Items for Systematic Reviews and Meta-analyses (PRISMA) guidelines (see Supplementary File 3 for Checklist).

\section{Eligibility criteria}

The population, intervention, comparison, outcome (PICO) framework was used to develop the inclusion and exclusion criteria for study selection in this review. The population of interest was any human study with participants aged between 0 and 100 years. Studies were excluded if the targeted populations are with rare diseases, defined as having a prevalence of 1 in 2000 persons [44]. Studies were also excluded if there was no index of SES status (e.g. SES index, income, education, employment) used to characterise the participants. Interventions were included if they adopted an RCT design (including cluster RCT) as we view this as the best way to identify causally valid and homogenous studies, and used a digital technology, which we operationalise as any web-based interface or wearable device that communicates information to the user, any mobile-based program, or offlinecomputer program, as the primary intervention tool. Studies were excluded if they contained a pharmacological component alongside the digital technology within an intervention, or if thegital technology was secondary to a therapeutic, face-to-face or counselling based intervention. As the focus of the review was on the effectiveness of digital technology, studies were only included if the comparator group did not receive any digital technologybased intervention. Lastly, studies that did not have any measure of PA (such as time in moderate to vigorous intensity, steps or sedentary time) as an outcome were excluded from the review.

\section{Search strategy}

A combination of terms relating to or describing the intervention was used to run the search. The search period was from January 1990 until March 2020 as it was assumed that any study which pre-dated this point in time would not be generalisable to our current understanding of digital interventions. All authors contributed to the development of the search strategy, and the full list of search terms has been provided in Supplementary File 1 . The search was conducted by an expert subject librarian using MEDLINE, Embase, PsycINFO, Web of Science (Science and Social Science Citation Index), Scopus and The Cochrane Library (Cochrane Database of Systematic Reviews, Cochrane Central Register of Controlled Trials (CENTRAL), Cochrane Methodology Register). The search terms within MEDLINE and Embase included a filter for controlled trials of interventions. Reference lists from relevant systematic reviews and metaanalyses were searched to identify any additional studies. Where relevant protocol papers were identified during the search, an attempt was made to find the accompanying trial papers. Only papers published or available in English language were considered.

\section{Data extraction}

Data were reviewed and extracted in pairs formed by five members of the team (MW, MA, II, KM, UJ). Initially, titles were checked for relevance and the abstracts of the 
relevant titles were screened. Partial data extraction was conducted from the full-texts of the relevant abstracts to assess the inclusion and exclusion criteria. Detailed data extraction was then conducted from the full texts of the included studies. At each stage, two authors independently reviewed the titles, abstracts and full-texts to include or exclude them for the next stage. Any disagreements were resolved via group discussion. With regards to research question 3, behaviour change techniques (BCTs), which were coded according to the comprehensive definitions found in Michie et al's BCT Taxonomy v1 [41], were extracted by two independent reviewers (MW and II) for each analysed study, with any disagreements resolved via group discussion with the full authorship team. The data extraction form can be found in Supplementary File 1.

\section{Data analysis}

Quantitative data for meta-analysis were identified either through extraction from published manuscripts, or through requesting additional summary statistics from authors, or by requesting individual participant data from authors and constructing our own summary statistics. The study team created a hierarchy of preferred metrics for both SES (1. Specific SES measure or index of deprivation; 2. Income; 3. Education; 4. Employment) and PA (1. minutes of Moderate-to-Vigorous-Intensity PA, 2. Total PA minutes, 3. Steps, 4. Sedentary time). Where studies reported multiple SES measures (e.g. education and income) or PA outcomes (e.g. steps and MVPA) in their manuscripts, a request to authors was made in line with the highest-ranking metric of interest in this hierarchy. Definitions for what constituted low and medium/ high SES was decided on a study-by-study basis based on the measures reported and what was appropriate for the context and study (for example country of origin and whether continuous or categorical scales were used to collect the data). For deprivation indices decile or quintile cut points were most often reported. For education most authors used a split between pre-university education and university educated or higher. Income splits differed by currency and year of publication, but a median household cut point was most frequently used. Employment was used more rarely (one study) and was split by manual or intermediate versus higher managerial. Table 1 indicates the specific definitions for each study. Authors of potentially relevant papers $(n=49)$ were contacted twice and given a minimum of 2 weeks each time to respond to requests for additional information. Thirty authors were either unable to provide data or did not respond to the request.

PA outcomes were extracted and combined using random-effects meta-analysis. This decision was made in advance of conducting meta-analysis and based on the expected heterogeneity in study designs, settings, interventions, populations, and time frames. Fixed effect meta-analysis is also reported as was pre-specified in our PROSPERO registration. Baseline and Follow-up scores in PA (or more rarely change scores) were extracted from papers or individual-level data. Measures of precision were extracted from standard errors, or standard deviations. For most of the studies included we were given access to the raw data and so we were able to calculate standard deviations directly. Where studies had multiple arms in their trial that were eligible for inclusion in the analysis, the control group was split equally between intervention arms to avoid double counting of participants.

The analysis for research questions 1 and 2 was performed in the $\mathrm{R}$ programming language and environment version 3.6.1 [64] and using the 'meta' package, [65]. $\mathrm{I}^{2}$ statistics were calculated for meta-analysis and forest plots produced. Where more than 10 studies were included in forest plots for our primary objectives a funnel plot was also produced to explore publication bias and Egger's statistic for assessing publication bias was calculated [66]. Research question 3 was addressed using meta-regression using the 'metareg' package in $\mathrm{R}$ (version 4.15-1) using a single explanatory covariate (number of behaviour change techniques employed at a study level) in order to explore whether studies employing more behaviour change techniques were observed to have larger intervention effects. Bubble plots were used to summarise the findings.

Sub-group analysis was pre-specified for the following categories: digital interventions with a sole focus on PA versus other targets (e.g. weight loss), study setting (countries/continents), excluding studies at high risk of bias, age groups (Under 5, 5-18, Adult 19-64, > 64 years), healthy or general population/versus chronic disease populations, duration of the active intervention (less than 3 months, 3-6months, greater than 6 months), duration of follow-up (less than 6 months, more than 6 months to 1 year, more than 1 year), and pregnancy. Cluster randomised trials had sample sizes adjusted to effective sample sizes accounting for average cluster sizes and Intracluster Correlation Coefficients (ICCs).

\section{Risk of bias assessment}

Two authors (MW and KM) performed an independent assessment of the risk of bias on each of the included studies in line with the updated Cochrane Risk of Bias in randomised trials 'RoB 2' [67]. All studies were graded by both authors with disagreements being resolved in discussion with the wider research team. The scoring algorithms presented in the RoB 2 were used to determine 


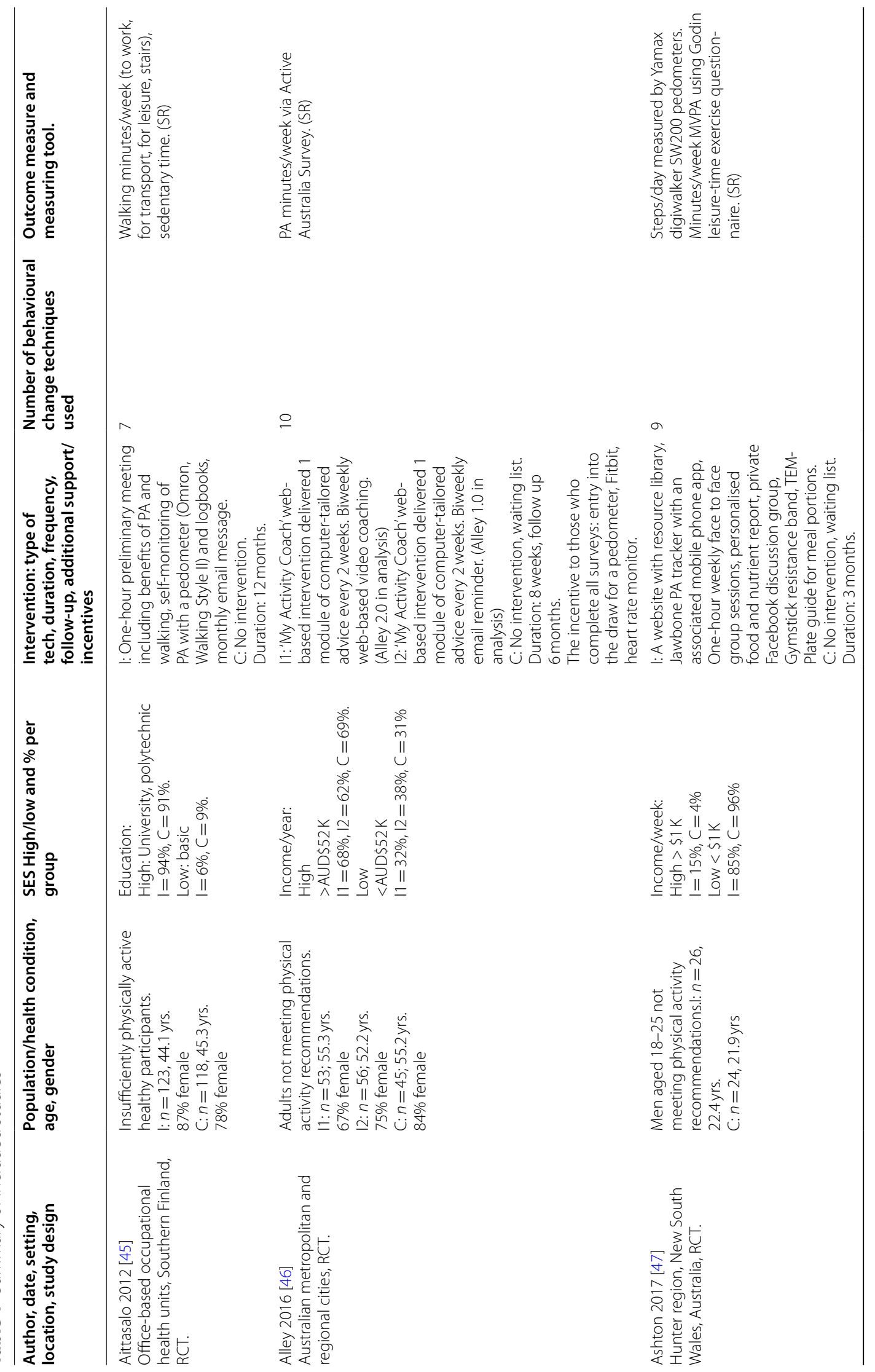




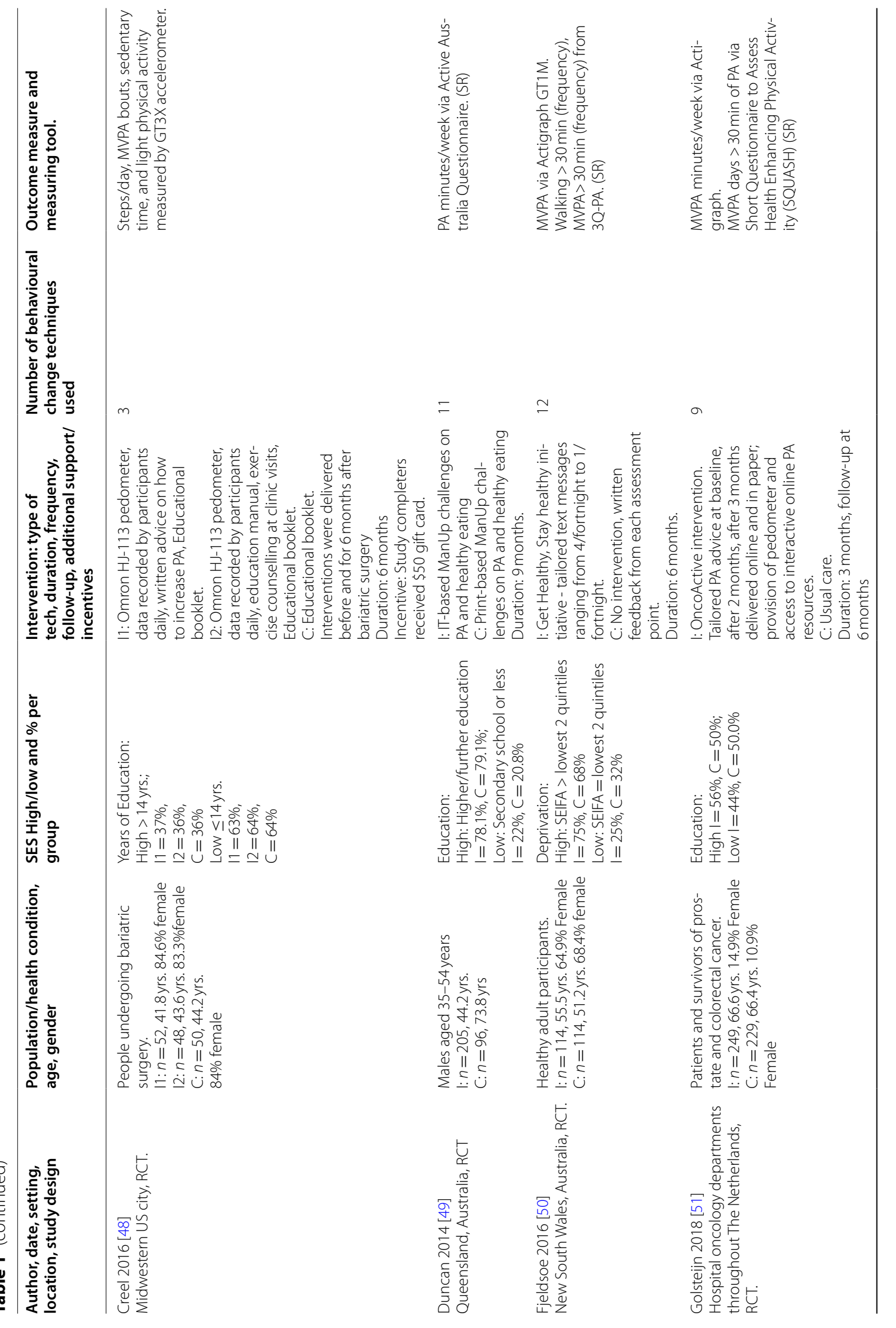




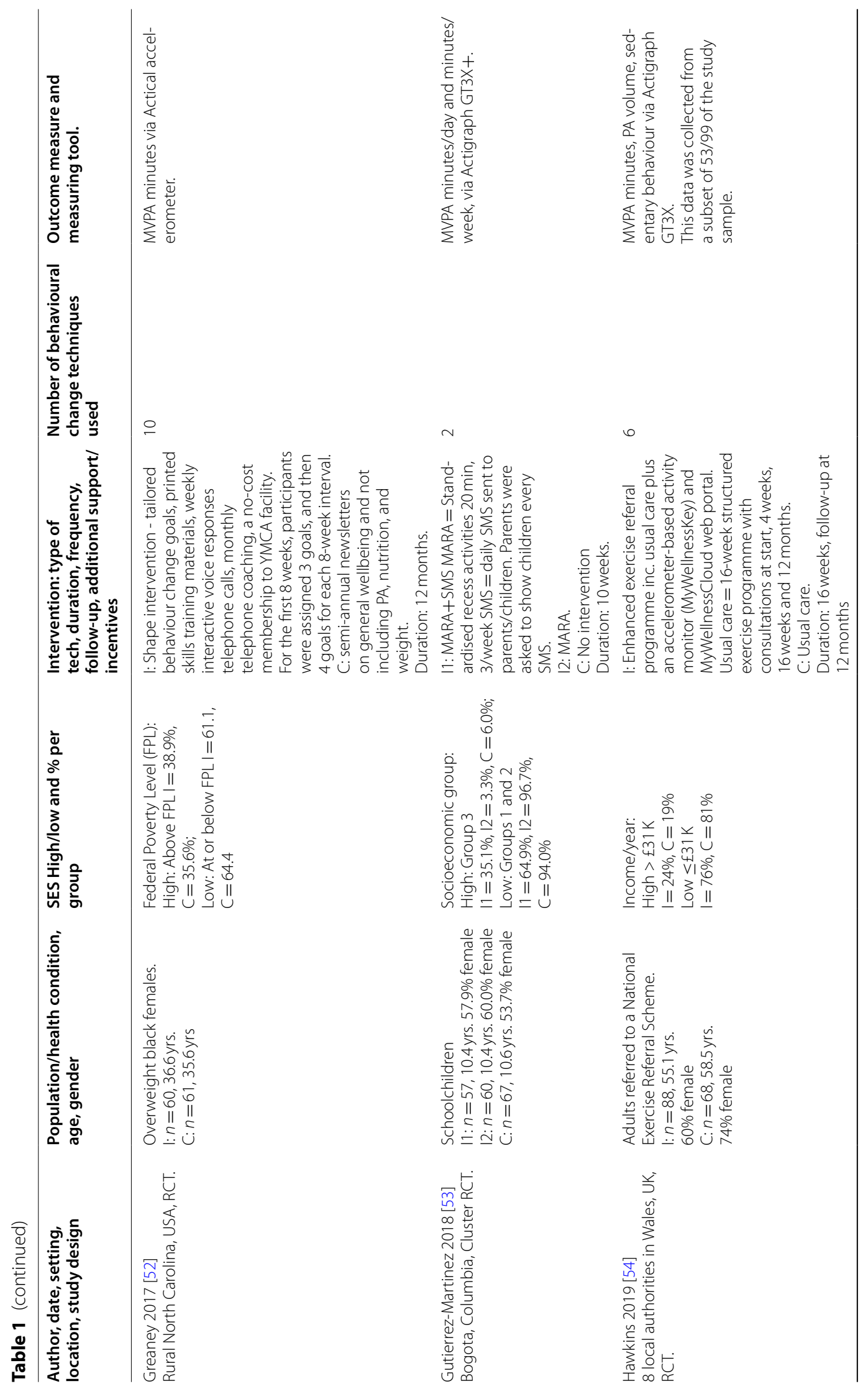




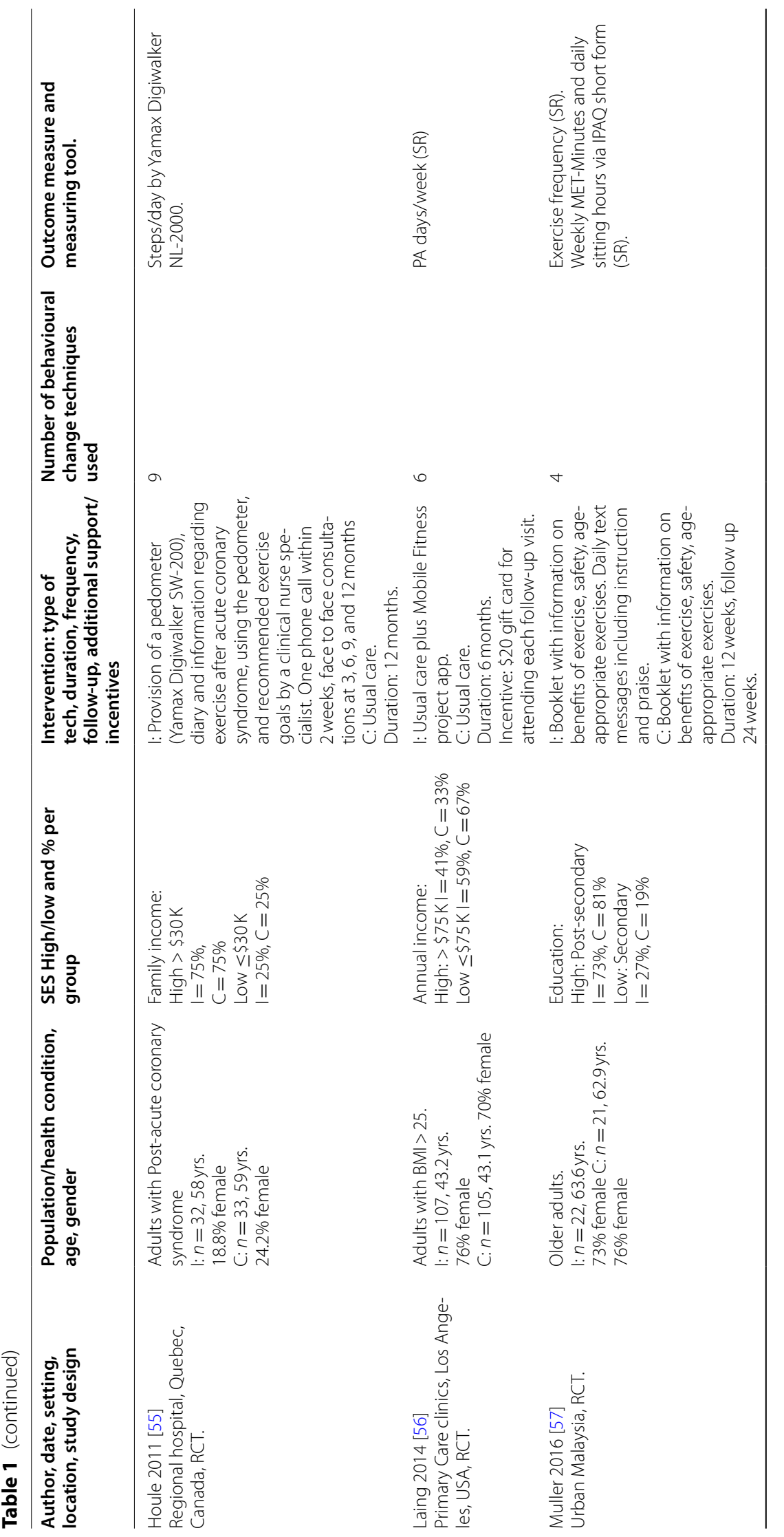




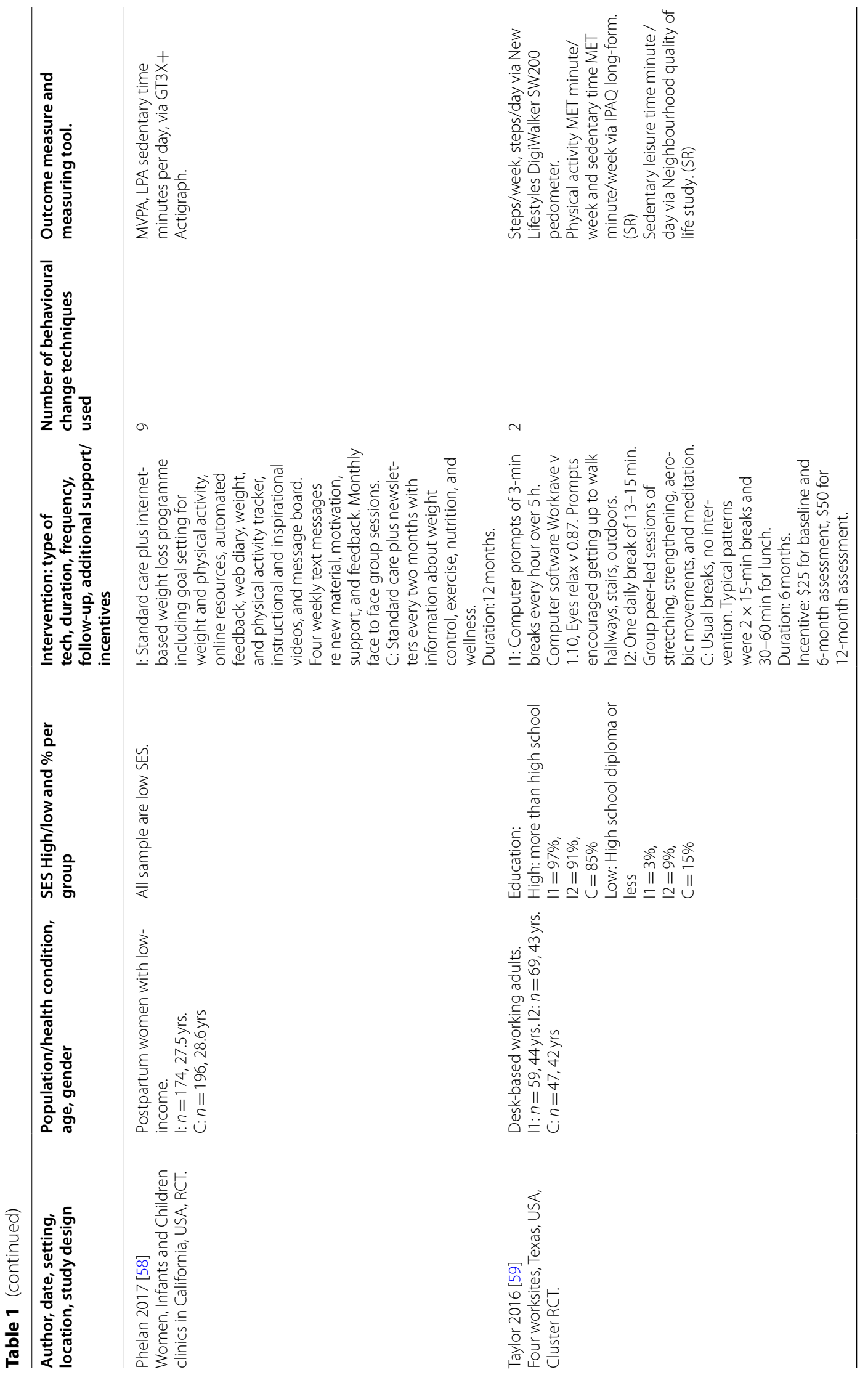




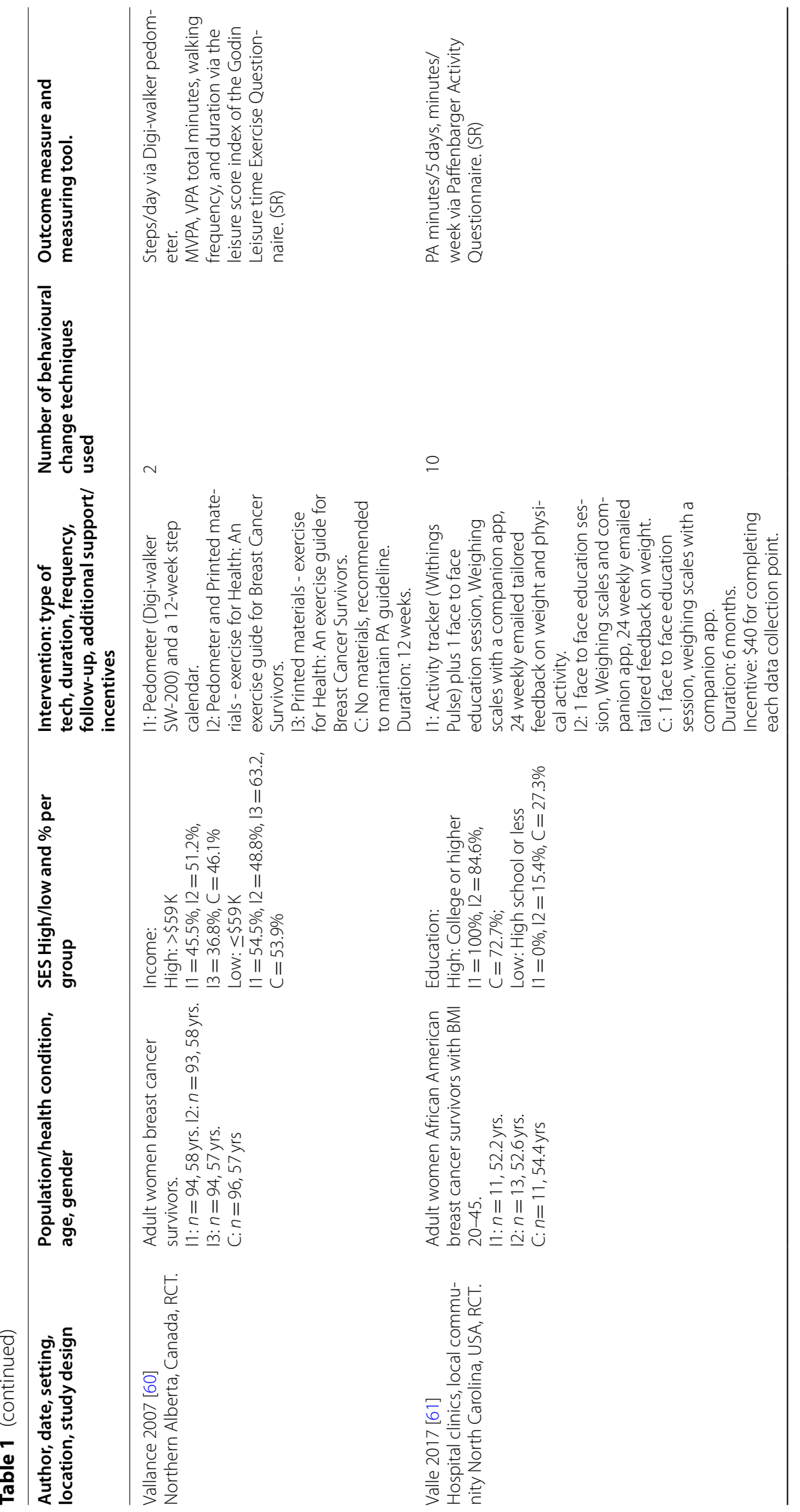




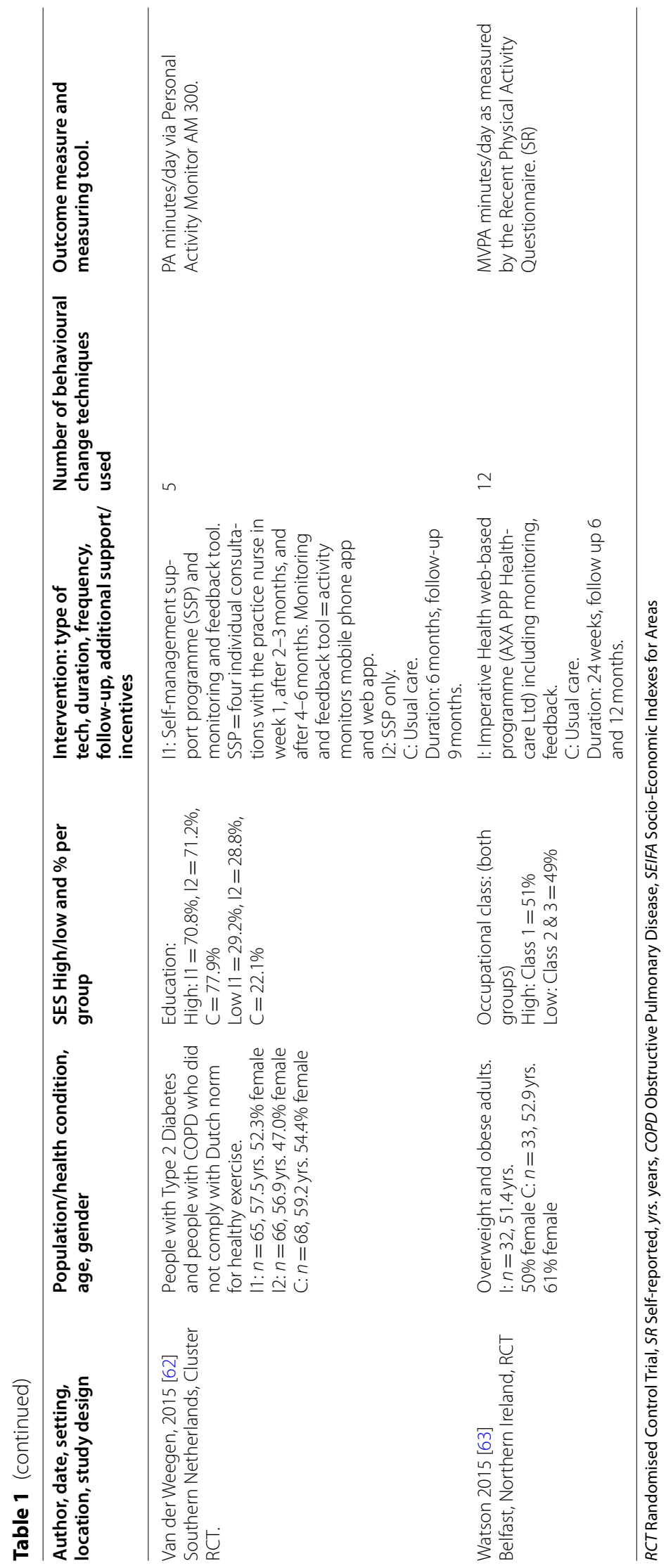


the low, moderate, or high risk of bias against each of the core six criteria.

\section{Results}

A PRISMA diagram for the study selection process including reasons for exclusion is shown in Fig. 1.

\section{Study characteristics}

The review included 19 studies comprising 16 RCT $[45-52,54-58,60,61,63]$ and three cluster RCT [53, $59,62]$, which are summarised in Table 1 . The studies took place in North America $(n=8)[48,52,55,56,58-$ 61], Europe $(n=5)[45,51,54,62,63]$, Australia $(n=4)$ [46, 47, 49, 50], Asia $(n=1)$ [57], and South America $(n=1)$ [53]. Eleven interventions were explicitly targeting PA behaviour $[45,46,48,51,53-55,57,59,60,62]$, while eight were targeting weight loss, general health or multiple lifestyle behaviours (e.g. PA and diet) [47,
$49,50,52,56,58,61,63]$. The included studies used a number and a combination of digital technologies such as web-sites $[46,47,49,51,54,58,59,62,63]$, activity trackers $[45,47,48,51,52,54,55,58,60-63]$, text messaging or email feedback or prompts $[45,46,50$, $53,57,58,61]$, and mobile applications $[47,49,56,61$, $62]$ in their interventions. Interventions lasted between 8 weeks and 12 months, most common durations were 6 months $(n=5)$ and 12 months $(n=4)$ as described in Table 1. Outcome measures included a range and combination of PA assessment methods (Table 1). Using our hierarchy for prioritising outcome measure, our primary analysis involved ten studies using selfreported measure of MVPA [51, 60, 63], total physical activity $[46,49,54,56,57,61]$ or walking [45], and nine studies used device-based assessments of MVPA [47, $48,50,52,53,58,62]$, leisure time PA [59], or steps [55] (Figs. 2 and 3).

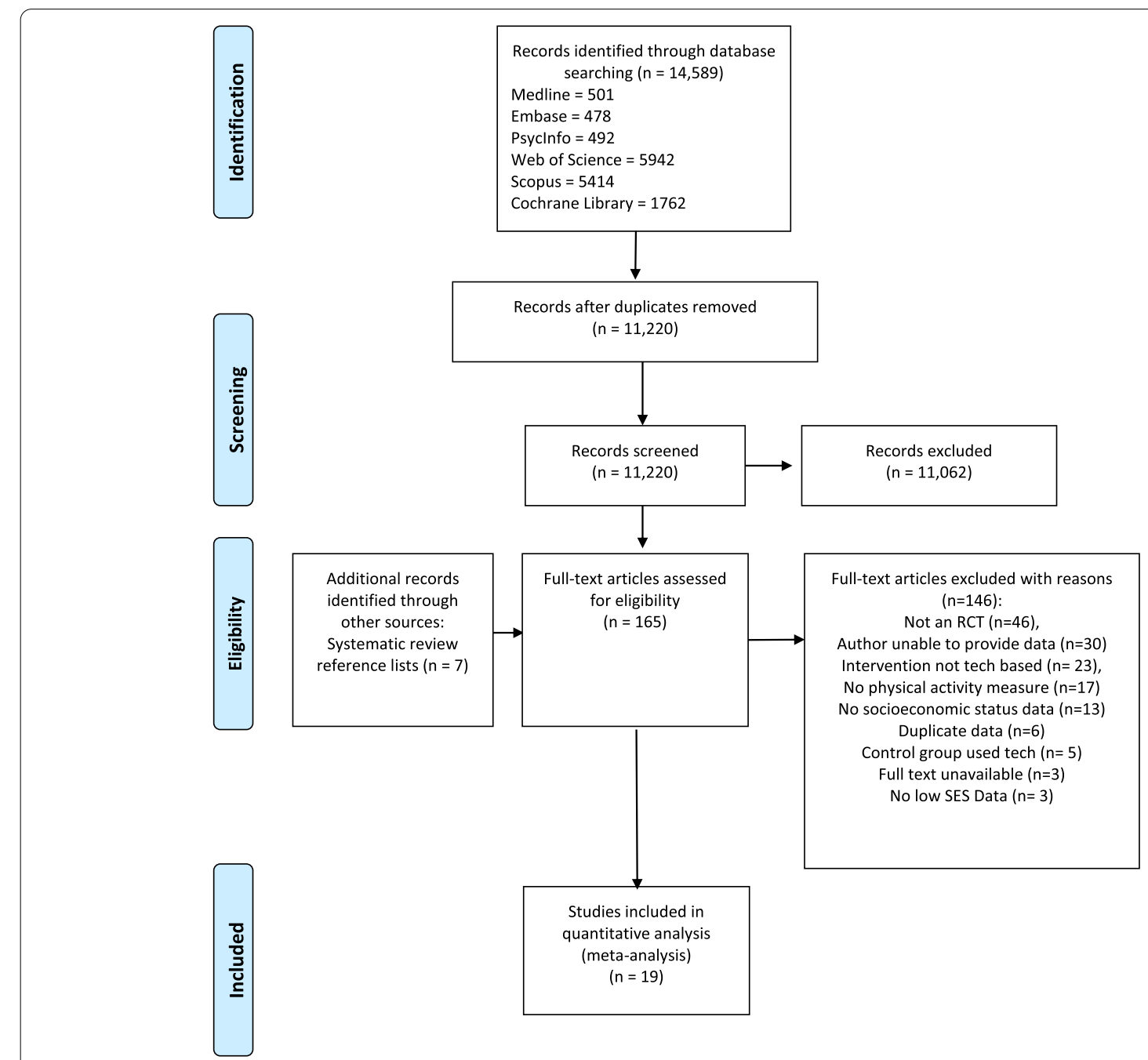

Fig. 1 PRISMA flow diagram of study selection 


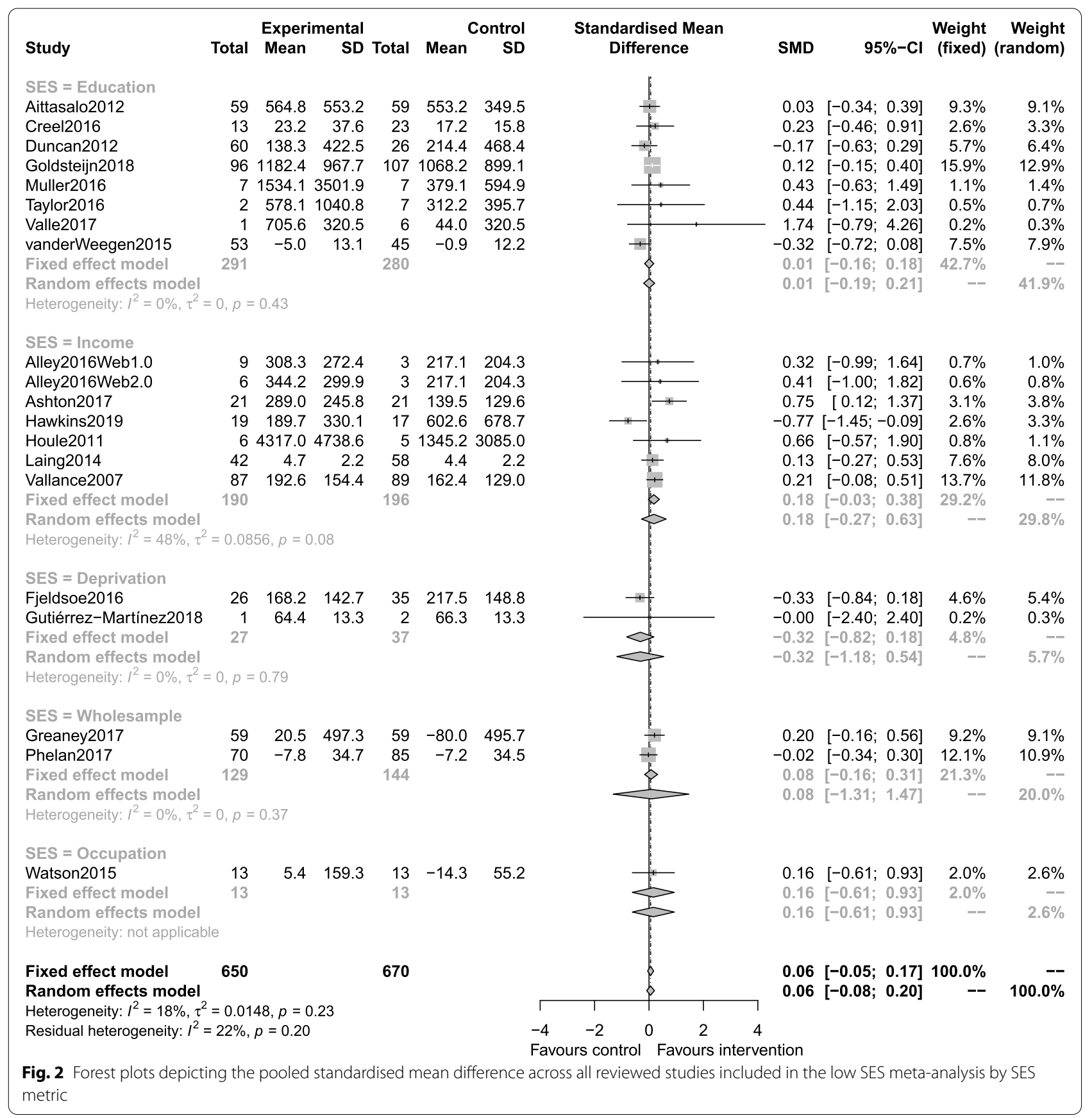

Measurement of SES was recorded in different ways across studies, with some studies including a number of methods. In the meta-analysis, the best measure of SES was considered from the following pre-specified priority list: deprivation score (i.e. index of multiple deprivations, SES group or federal poverty line, $n=3[50,52$, $53])$, income $(n=6[46,47,54-56,60])$, education $(n=9$ $[45,48,49,51,57-59,61,62])$, employment ( $n=1[63])$. Each SES was then dichotomised into date-adjusted high and low categories (Table 1). Socio-Economic Indexes for Areas was categorised as low if in the lower two quintiles. Federal poverty level was categorised as low if at or below the Federal Poverty Line. The median income for the specific country at the time of data collection was used as a cut-off between high and low income. Education was low if equivalent to 14 years or less (i.e. no higher education). SES group was categorised as low for the two lowest groupings used. While the included studies randomised 


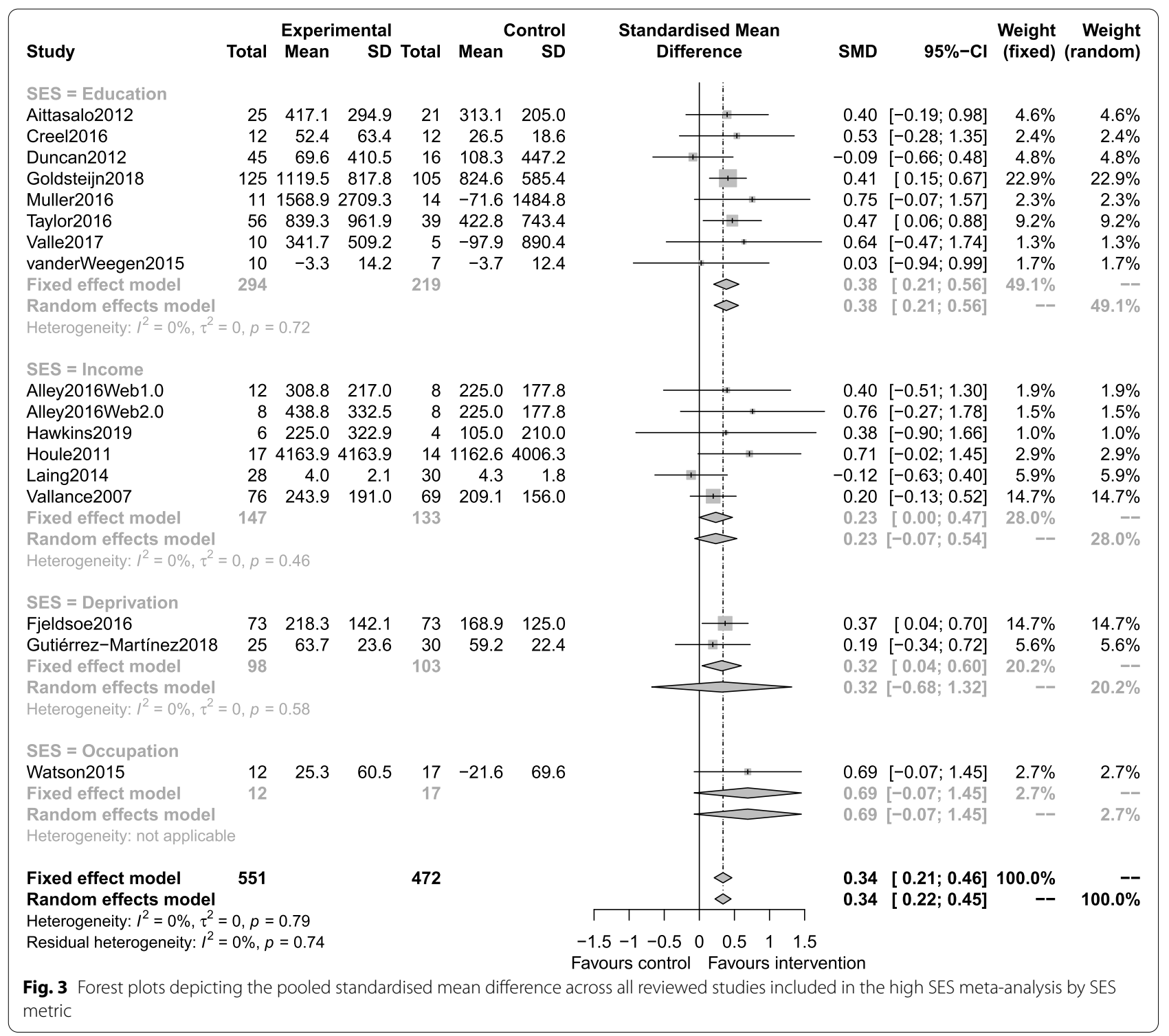

5419 participants between them, once we had accounted for attrition, data availability and a focus on relevant study arms we ended up with a sample of $n=1317$ low SES and $n=1023$ medium-high SES participants for analysis.

The three cluster randomised trials had their sample sizes scaled according to their average cluster sizes. Only one study [62] reported an ICC (of 0.005) and this was used for all three studies.

\section{RQ1 effectiveness of digital interventions on physical activity in low socioeconomic status groups}

Twenty interventions from nineteen studies were included in this meta-analysis (one study, Alley et al., 2016 [46], appears twice as it was a three-arm trial).
Interventions were grouped according to how they measured SES. Heterogeneity was low $\left(\mathrm{I}^{2}=18 \%\right)$. There was little difference between the fixed and random-effects analysis. This analysis did not identify a statistically significant intervention effect in low SES groups (standardised random-effects estimate: $0.06,95 \%$ CI $[-0.08,0.20]$ ). A funnel plot did not indicate publication bias $(p=0.37)$.

\section{RQ2 equivalence of digital interventions on physical activity in low socioeconomic status groups}

Seventeen interventions from sixteen studies were included in this meta-analysis conducted in high SES participants (again Alley 2016 appears twice, with a split control group), split by how SES was determined. Heterogeneity was low $\left(\mathrm{I}^{2}=0 \%\right)$. Fixed and random-effects 


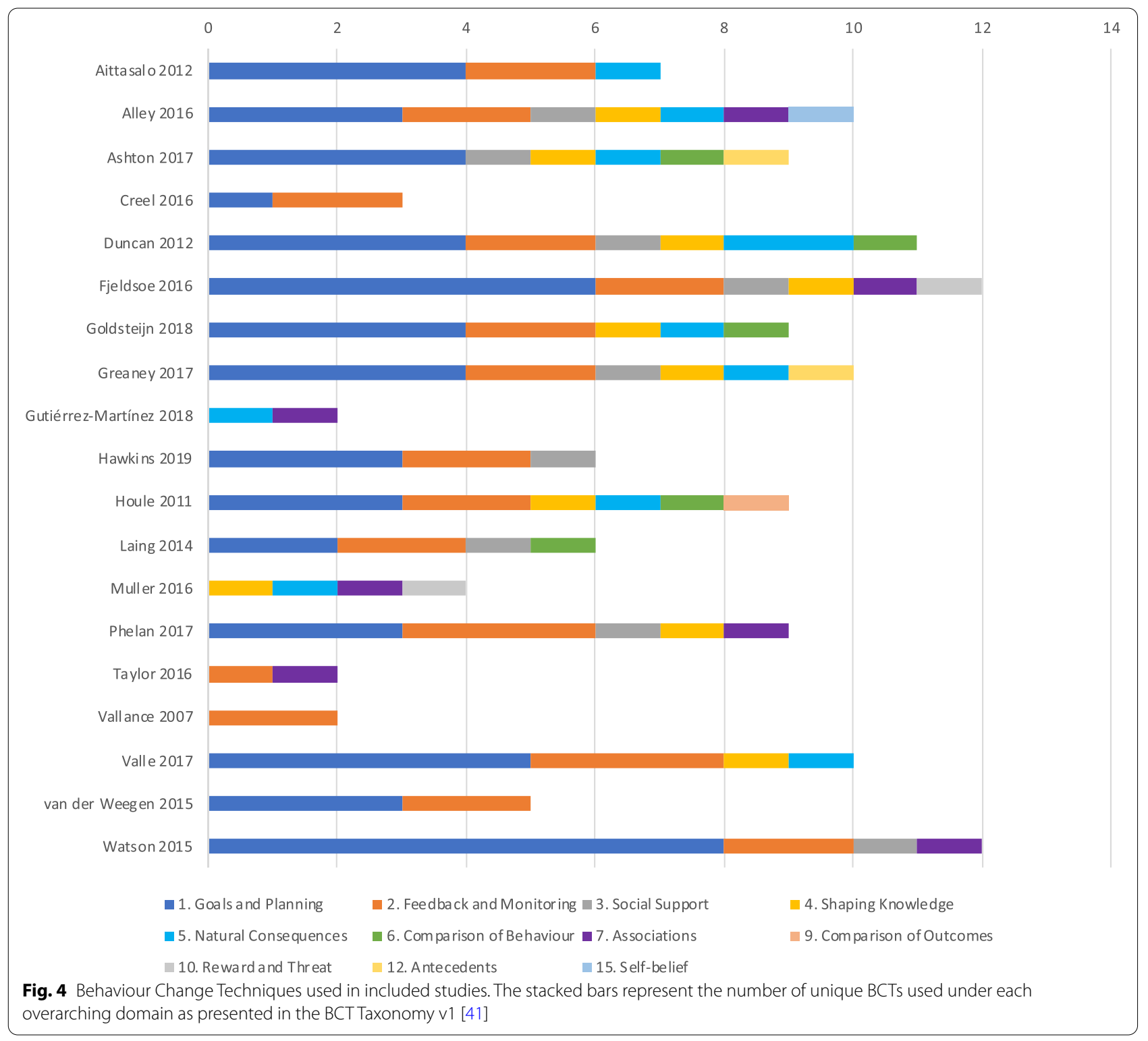

estimates did not differ substantially. This analysis identified a statistically significant effect of about a third of a standard deviation in favour of intervention for this group (standardised random-effects estimate: 0.34, 95\% CI $[0.22,0.45])$. A funnel plot did not indicate publication bias $(p=0.45)$.

\section{Subgroup analysis for RQ1 and RQ2}

We were able to conduct subgroup analysis for some of our pre-specified categorisations. These were: digital interventions with a PA only focus, country, chronic disease, and intervention length. We were not able to explore studies at high risk of bias (only two studies at high risk of bias provided data), age group (included age groups were too disparate), and pregnancy (only one study had a postpartum focus). Post-hoc subgroup analysis was also performed to explore whether there were any differences depending on objective versus self-report measures of PA, and whether the study used and active or passive control condition. None of our subgroup analyses indicated differential effects by subgroup (Supplementary File 2, Appendices A and C).

\section{RQ3 what behaviour change techniques are most effective in low SES groups?}

Figure 4 displays the BCTs found in each intervention. The reviewed studies used a mean of 7 BCTs (range $=2-12$ ). The most common BCTs were 


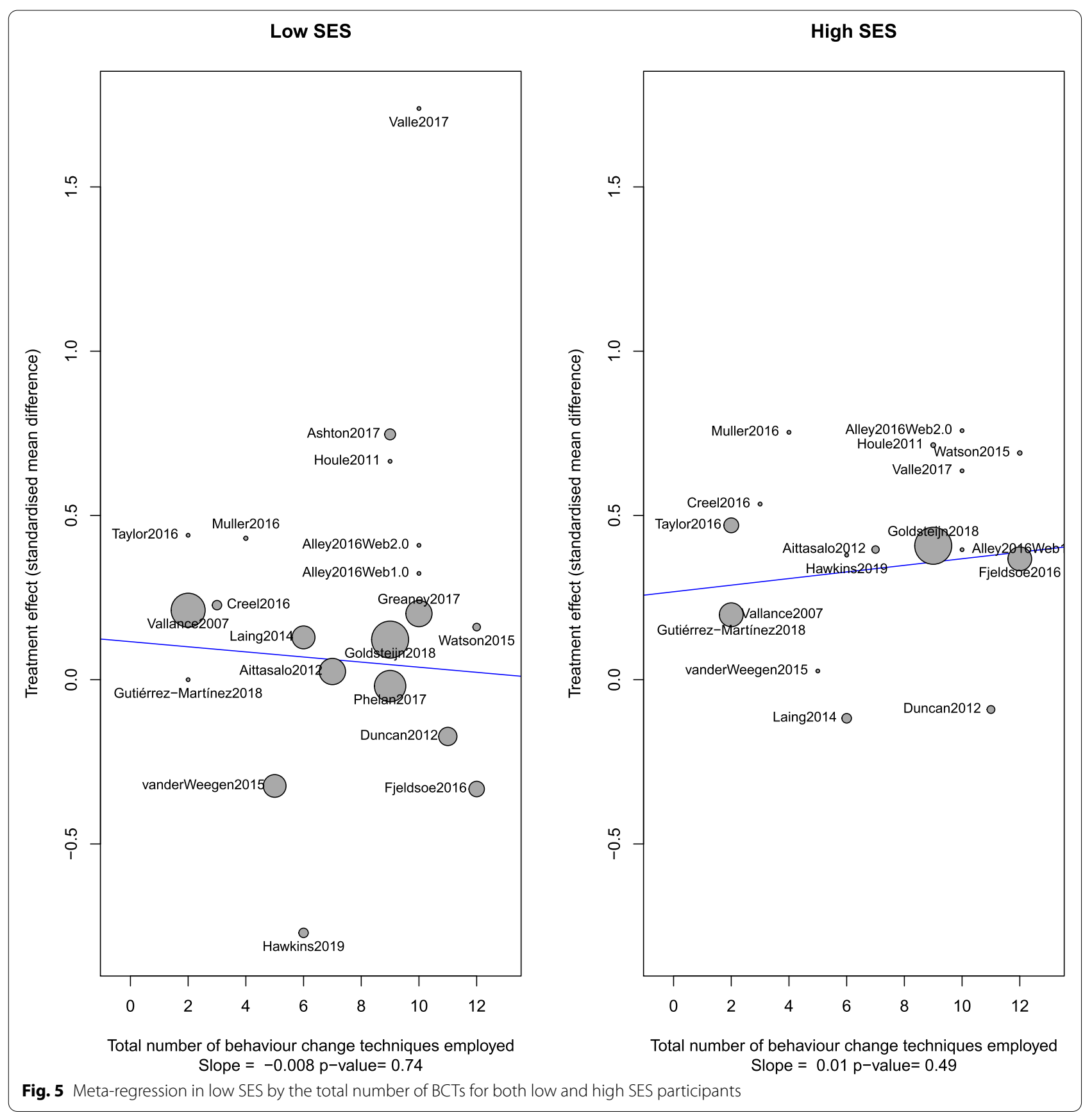

Self-monitoring of behaviour (81\%), Goal setting behaviour (76\%), Feedback (76\%), Problem-solving (52\%), Action planning (52\%), Information about health consequences (48\%), Behaviour goal review (43\%) and Social support (43\%). Post-hoc meta-regression of the number of BCTs employed by each study revealed no statistically significant trend between the amount of BCTs employed for either low or high SES groups (Fig. 5). Subgroup analysis of individual BCTs with more than one constituent indicator (goals and planning, feedback and monitoring, shaping knowledge, natural consequences, comparison of behaviour, reward and threat, and antecedents) did not indicate sub-group effects.

\section{Risk of bias}

All 19 of the included studies were assessed for risk of bias (Fig. 6). Four studies were considered low risk of 


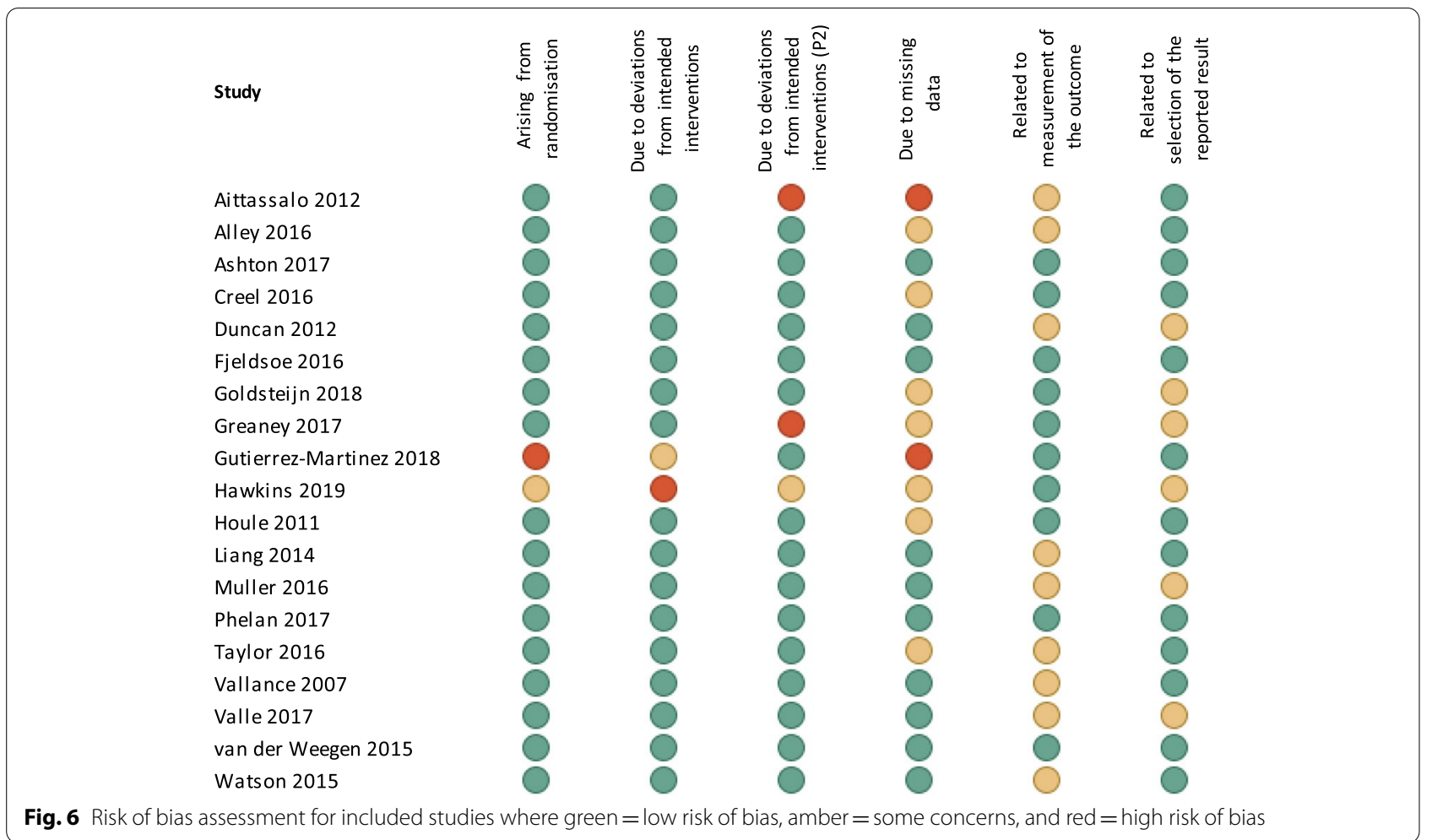

bias for all categories, four had one category judged to have some concerns, six had two categories judged to have some concerns and four had at least one category judged as high risk of bias. Given the behavioural nature of the trial, blinding to allocation was not possible in any of the studies, and a distinction was made in terms of outcome measure being self-report vs. device-based, with the latter considered to incur less risk than the patient-reported former.

\section{Discussion}

In this systematic review and meta-analysis, we provide evidence that digital behaviour change interventions aimed at increasing PA are effective for people of high SES but were not observed to be beneficial for people of low SES. In particular, our analysis of 19 studies with 20 interventions found no evidence of effect in sub-samples defined as low SES, but a statistically significant, smallto-medium effect size in high SES participants. This effect was consistently observed across SES indicators, geographical setting, the clinical status of the population, length of intervention, and PA assessment method. Most studies used self-regulatory BCTs such as self-monitoring, goal setting and feedback as the primary intervention features, but the number nor type of BCTs used in interventions were associated with the outcome in high or low
SES particpants. The studies included in this review were mostly of moderate or low risk of bias.

This, to our knowledge, is the first systematic review and meta-analysis that has analysed digital interventions targeting PA behaviour according to SES. Other systematic reviews and meta-analyses have looked at digital interventions without stratifying by SES. Stockwell et al. observed a pooled standardised mean difference in PA of 0.28 across 8 RCTs of digital behaviour change interventions targeting PA in older adults [68]. Davies et al. looked at internet-delivered PA interventions in adults demonstrating a pooled effect size of 0.16 across 25 studies that used an RCT design [69]. Most recently, Laranjo and colleagues demonstrated a pooled standardised mean difference of 0.35 in their meta-analysis of $28 \mathrm{RCTs}$ involving 7454 adults that underwent mobile application or activity tracker-based interventions [70]. The discrepancy in effect size observed between high and low SES in the present study may give some indication that the net benefit observed in these comparative reviews could be driven by a higher proportion of high SES research participants within the reviewed studies.

In this review, there was no indication that any methodological differences such as study duration, PA outcome measure, SES metric, country, or health status of the target population between studies had any impact on the findings. The application of digital technology varied 
considerably between the reviewed studies, ranging from motivational text messaging, feedback from wearable activity tracking devices, and sophisticated, multi-component, web-based interventions. The common BCTs used within these studies are akin to those found in commercial and research-based digital behaviour change tools targeting PA [71-73]. While the evidence base in favour of using digital technologies containing self-regulatory BCTs is growing $[70,74,75]$, our results suggest that such interventions may be of little benefit to participants of low SES irrespective of complexity (i.e. number of $\mathrm{BCTs}$ ) content (i.e. type of BCTs). Put another way, although access to these technologies may be improving, simply receiving interventions, even those that are efficacious in the more educated and recurrently researched higher SES populations, may not provide adequate support to those who are more deprived, less educated and/ or have lower income. Consequently, more research into the BCTs that serve lower SES populations is needed.

Of course, simply receiving an intervention does not guarantee effective engagement with that intervention in a way that leads to behaviour change [76]. One important aspect we were unable to tease out from the reviewed studies is whether the dose of intervention received and the utilisation of key intervention features or BCTs were equal between high and low SES participants. Future studies deploying digital interventions for promoting PA would do well to monitor and report meaningful usage and engagement to see if this is equivalent between the low and high SES participants [77]. Additionally, people of low SES may, in general, tend to use the internet less for health information and have a lower eHealth literacy, i.e. people's capability to use information and communication technology to improve their health, which may impact intervention engagement [30, 34, 78]. Levels of eHealth literacy are positively associated with lifestyle behaviour $[79,80]$. In the context of digital PA interventions, eHealth literacy might translate as the users' ability to navigate the technological devices themselves, understand the information received from the educational components, and appropriately apply the self-regulatory $\mathrm{BCTs}$ that are advocated. Incorporating intervention components that identify low eHealth literacy and boost it as a preliminary objective prior to implementing behavioural support may be one way of making these interventions more equitably beneficial.

Another possible explanation might be that the antecedents of PA may vary between people of low and high SES [81]. Pertinent frameworks of behaviour suggest that individuals need to have the capability, opportunity and motivation to be able to make changes [82]. Compared to those of low SES, people of higher SES may elicit more opportunities to act upon intervention advice or feedback through more free time, the ability to prioritise lifestyle behaviour and more resources, as well as a more supportive social and physical environment that facilitates increases in PA [83]. A range of behavioural theories was used to inform the interventions used in the reviewed studies, but it is unclear if the application of these theories was tailored in any way to meet the needs of study participants with varying SES, demographics and circumstances. As the importance of personalisation of digital behaviour change tools is increasingly recognised and tailored interventions are being implemented [76, 84, 85], ensuring that contextual factors related to SES that may influence behaviour are catered for would be a useful direction for further intervention research.

In light of the findings of this review and the acceleration towards a digital world (escalated by the COVID19 pandemic), there is an urgent need to investigate whether digital behaviour change interventions are widening rather than reducing inequalities [86]. Our review looks at the equivalence of effect on PA behaviours, but it would not be unreasonable to assume that similar findings would be observed in research targeting other health or behavioural outcomes. Investigating whether these technologies can benefit people of low-SES, and how to improve their efficacy for this sub-population who are invariably the most in need of lifestyle support, should be a public health priority. Inevitably, developers of commercial technology for supporting PA behaviour may not prioritise lower SES segments should the goal be to maximise revenue, so the onus will likely be on researchers and public health advocates to address the discrepancy in the effectiveness of digital interventions between SES groups.

Investing in research and development for technologies that explicitly support PA among low SES populations could be a valuable public health strategy given the potential for maximising reach in populations who disproportionately utilise healthcare resources. There are certainly ways that the research community could augment progress in this area: by better reporting the SES component of their sample across multiple indices and making a concerted effort to recruit people of lower SES to their research trials so that more extensive evaluation of this sub-population can be conducted. When developing digital interventions targeting PA or other lifestyle behaviours, researchers should adopt a personcentred approach $[87,88]$ that encourages the use of guiding principles to ensure that the design features meet the needs and context for all individuals across the SES spectrum. Similarly, creating digital resources using participatory research which targets low SES users will help to ensure that the most pertinent $\mathrm{BCTs}$ and features are 
used in a way that will enhance engagement and the likelihood of behaviour change [89].

The key strengths of this systematic review are the comprehensive literature searching, screening, data extraction and risk of bias assessments, as well as the retrieval and analysis of raw individual level or summary data from studies regarding the SES measures from exclusively RCTs. We also observed low statistical heterogeneity $\left(\mathrm{I}^{2}\right)$ scores in our meta-analyses, indicating a robust analytical approach when determining our pooled effect sizes.

Conversely, a limitation of this study is the high methodological heterogeneity of included studies. The variability in the SES metric, which used different constructs on varying ordinal or continuous scales, made standardising a 'low SES' definition or threshold across studies challenging. While every effort was made to take a systematic, time-referenced approach, it must be acknowledged that the grouping of low SES participants may not be equivalent from one study to the next. Our broad definitions of digital health and PA meant that intervention characteristics and outcome measures were variable across studies with some including more digital or nondigital components than others, which makes unpacking the specific mechanisms that drive the findings difficult in the present study. Similarly, the control groups included in the review differed from one study to the next, which could explain the lack of observed effect in low SES groups, although it should be acknowledged that this would not explain the discrepancy in effects observed within a study between low and high SES participants. A further limitation is the searching of literature, which, by solely targeting research databases and articles written in the English language, may not have included all available research on this topic. Similarly, there were eligible articles for which study authors were unable to provide the necessary stratified data and further titles that will have been published following the analysis and publication of the present systematic review.

\section{Conclusions}

In this study, we demonstrate that, at present, digital interventions targeting PA are not equivalently effective for people of low and high SES. Specifically, there is very little evidence that digital PA interventions have any efficacy for people of low SES, but moderate efficacy for those of high SES, both between and within studies. Increasing access to information communication and wearable technology amongst even the most vulnerable people has led to digital interventions being championed as a tool for reducing inequalities in health promotion. This study suggests that in a PA context the opposite is true, that is, people who would benefit the most from these interventions are being left behind. We recommend that future development of digital interventions aimed at improving PA must make more effort to meet the needs of low SES people within the target population.

\section{Supplementary Information}

The online version contains supplementary material available at https://doi. org/10.1186/s12966-021-01218-4.

\section{Additional file 1.}

Additional file 2.

Additional file 3.

\section{Acknowledgements}

We would like to extend a big thank you to all of the corresponding authors of the studies we included in this review and their co-author teams for providing the data that enabled us to conduct our analysis. We would also like to thank Delyth Morris, Medical Subject Librarian and Ms Mala Mann, Systematic Review Consultant at Cardiff University, for helping us to put the literature search together. During the course of this project the team welcomed four babies to the world and their contributions must be acknowledged: thanks to Joseph Kelson, Alexander Armstrong, Orla Western and Cara Kelson. Without your input the review would have been done in half the time.

\section{Authors' contributions}

All authors conceived and designed the study and developed the search strategy. II conducted the search, with input from a subject librarian. MW, MA, II, KM and UJ screened studies for inclusion and extracted data. MW and KM analysed studies for Risk of Bias, MA prepared the PRISMA data, UJ elicited the study characteristics and MW and II coded behaviour change techniques from the reviewed studies. MK analysed all data and all authors interpreted the results. MW drafted the manuscript with all co-authors contributing to drafts of the paper. All authors approved the final manuscript.

\section{Funding}

The research team received seed funding from the GW4 Alliance (https:// gw4.ac.uk) via Cardiff University mHealth Network collaboration to start the project. MW received a fellowship from the GetAMoveOn Network+ (EPSRC Grant ref.: EP/N027299/1), the activities of which contributed to his drafting of the manuscript. MA has been supported by grants from the UK Centre for Ageing Better, the Elizabeth Blackwell Institute, the Medical Research Council, and from the University of Bristol, outside the submitted work. KM was funded by a Health and Care Research Wales Health Fellowship Award [grant number HF-16-1164] and her work was undertaken with the support of The Centre for the Development and Evaluation of Complex Interventions for Public Health Improvement (DECIPHer), a UKCRC Public Health Research Centre of Excellence. Part of MK's time was funded by a fellowship from the Alan Turing Institute. Neither the GW4 Alliance or any funders of the authors time had any input on the design or execution of this review, nor the writing of the manuscript.

\section{Availability of data and materials}

All data and code to replicate our analysis are freely available at https://github. com/MarkKelson/REPAID

\section{Declarations}

Ethics approval and consent to participate

Not applicable.

\section{Consent for publication}

Not applicable.

\section{Competing interests}

The authors declare that they have no competing interests. 


\section{Author details}

${ }^{1}$ Centre for Motivation and Health Behaviour Change, Department for Health, University of Bath, Claverton Down, Bath BA2 7AY, UK. ${ }^{2}$ Centre for Exercise, Nutrition and Health Science, School for Policy Studies, University of Bristol, 8 Priory Road, Bristol BS8 1TZ, UK. ${ }^{3}$ PRIME Centre Wales, School of Medicine, Cardiff University, Cardiff CF14 4YS, UK. ${ }^{4}$ Centre for Development, Evaluation, Complexity and Implementation in Public Health Improvement (DECIPHer), School of Social Sciences, Cardiff University, Cardiff CF10 3BD, UK. ${ }^{5}$ School of Healthcare Sciences, College of Biomedical and Life Sciences, Cardiff University, Cardiff CF14 4XN, UK. ${ }^{6}$ Department of Mathematics/Institute of Data Science and Artificial Intelligence, University of Exeter, Laver Building, Exeter EX4 4QE, UK.

\section{Received: 28 February 2021 Accepted: 20 October 2021} Published online: 09 November 2021

\section{References}

1. 2018 Physical Activity Guidelines Advisory Committee. 2018 physical activity guidelines advisory committee scientific report; 2018.

2. World Health Organization. WHO guidelines on physical activity and sedentary behaviour: at a glance; 2020.

3. Rhodes RE, et al. Physical activity: health impact, prevalence, correlates and interventions. Psychol Health. 2017;32(8):942-75.

4. Bull FC, et al. 2020 guidelines on physical activity and sedentary behaviour. Br J Sports Med. 2020;54(24):1451.

5. Ding $D$, et al. The economic burden of physical inactivity: a global analysis of major non-communicable diseases. Lancet. 2016;388(10051):1311-24.

6. Guthold R, et al. Worldwide trends in insufficient physical activity from 2001 to 2016: a pooled analysis of 358 population-based surveys with 1\&\#xb7;9 million participants. Lancet Glob Health. 2018;6(10):e1077-86.

7. Baker EH. Socioeconomic status, definition. The Wiley Blackwell encyclopedia of health, illness, behavior, and society; 2014. p. 2210-4.

8. Braveman P, Tarimo E. Social inequalities in health within countries: not only an issue for affluent nations. Soc Sci Med. 2002;54(11):1621-35.

9. WHO. Global Health Observatory (GHO) data: life expectancy; 2016.

10. Marmot M. Social determinants of health inequalities. Lancet. 2005;365(9464):1099-104.

11. Mackenbach JP. The persistence of health inequalities in modern welfare states: the explanation of a paradox. Soc Sci Med. 2012;75(4):761-9.

12. Scholes S. Health survey for England 2016: physical Actviity in adults, N. digital, editor; 2017.

13. Cotter KA, Lachman ME. No strain, no gain: psychosocial predictors of physical activity across the adult lifespan. J Phys Act Health. 2010;7(5):584-94.

14. O'Donoghue $\mathrm{G}$, et al. Socio-economic determinants of physical activity across the life course: a "DEterminants of Dlet and physical ACtivity" (DEDIPAC) umbrella literature review. PLoS One. 2018;13(1):e0190737.

15. Beenackers MA, et al. Socioeconomic inequalities in occupational, leisuretime, and transport related physical activity among European adults: a systematic review. Int J Behav Nutr Phys Act. 2012;9(1):116.

16. Chastin SFM, et al. Inequality in physical activity, global trends by income inequality and gender in adults. Int J Behav Nutr Phys Act. 2020;17(1):142.

17. Samitz G, Egger M, Zwahlen M. Domains of physical activity and all-cause mortality: systematic review and dose-response meta-analysis of cohort studies. Int J Epidemiol. 2011:40(5):1382-400.

18. Lear SA, et al. The effect of physical activity on mortality and cardiovascular disease in 130000 people from 17 high-income, middle-income, and low-income countries: the PURE study. Lancet. 2017;390(10113):2643-54.

19. Müller AM, et al. Physical activity, sedentary behavior, and diet-related eHealth and mHealth research: Bibliometric analysis. J Med Internet Res. 2018;20(4):e122.

20. Kreps GL, Neuhauser L. New directions in eHealth communication: opportunities and challenges. Patient Educ Couns. 2010;78(3):329-36.

21. World Health Organization. Classification of digital health interventions v1. 0: a shared language to describe the uses of digital technology for health: World Health Organization; 2018.

22. Hootsuite and We Are Social. Digital 2020: global digital overview; 2020.
23. Statista. Telecommunications. Technology \& Telecommunications 2021; Available from: https://www.statista.com/markets/418/topic/481/telec ommunications/\#overview.

24. Fanning J, Mullen PS, McAuley E. Increasing physical activity with Mobile devices: a meta-analysis. J Med Internet Res. 2012;14(6):e161.

25. Lyons JE, et al. Behavior change techniques implemented in electronic lifestyle activity monitors: a systematic content analysis. J Med Internet Res. 2014;16(8):e192.

26. Krebs P, Prochaska JO, Rossi JS. A meta-analysis of computer-tailored interventions for health behavior change. Prev Med. 2010;51(3-4):214-21.

27. Bradbury K, et al. Developing digital interventions: a methodological guide. Evid Based Complement Alternat Med. 2014;2014:561320.

28. Mackert $M$, et al. Health literacy and health information technology adoption: the potential for a new digital divide. J Med Internet Res. 2016;18(10):e264.

29. Latulippe K, Hamel C, Giroux D. Social health inequalities and eHealth: a literature review with qualitative synthesis of theoretical and empirical studies. J Med Internet Res. 2017;19(4):e136.

30. Norman CD, Skinner HA. eHealth literacy: essential skills for consumer health in a networked world. J Med Internet Res. 2006;8(2):e9.

31. Bodie GD, Dutta MJ. Understanding health literacy for strategic health marketing: eHealth literacy, health disparities, and the digital divide. Health Mark Q. 2008;25(1-2):175-203.

32. Azzopardi-Muscat N, Sørensen K. Towards an equitable digital public health era: promoting equity through a health literacy perspective. Eur J Pub Health. 2019;29(Supplement_3):13-7.

33. Guo Z, et al. Socioeconomic disparities in eHealth literacy and preventive behaviors during the COVID-19 pandemic in Hong Kong: cross-sectional study. J Med Internet Res. 2021;23(4):e24577.

34. Neter E, Brainin E. eHealth literacy: extending the digital divide to the realm of health information. J Med Internet Res. 2012;14(1):e19.

35. Kontos E, et al. Predictors of eHealth usage: insights on the digital divide from the health information National Trends Survey 2012. J Med Internet Res. 2014;16(7):e172.

36. Jahangiry $L$, et al. Web-based physical activity interventions: a systematic review and meta-analysis of randomized controlled trials. Public Health. 2017;152:36-46.

37. McIntosh JRD, et al. Do E-health interventions improve physical activity in young people: a systematic review. Public Health. 2017;148:140-8.

38. Muellmann S, et al. Effectiveness of eHealth interventions for the promotion of physical activity in older adults: a systematic review. Prev Med. 2018;108:93-110.

39. Tang MSS, et al. Effectiveness of wearable trackers on physical activity in healthy adults: systematic review and meta-analysis of randomized controlled trials. JMIR Mhealth Uhealth. 2020;8(7):e15576.

40. Cotie LM, et al. The effectiveness of eHealth interventions on physical activity and measures of obesity among working-age women: a systematic review and meta-analysis. Obes Rev. 2018;19(10):1340-58.

41. Michie $\mathrm{S}$, et al. The behavior change technique taxonomy ( $\mathrm{v} 1$ ) of 93 hierarchically clustered techniques: building an international consensus for the reporting of behavior change interventions. Ann Behav Med. 2013;46(1):81-95.

42. Michie $\mathrm{S}$, et al. Effective techniques in healthy eating and physical activity interventions: a meta-regression. Health Psychol. 2009;28(6):690-701.

43. Whatnall MC, et al. Effectiveness of interventions and behaviour change techniques for improving physical activity in young adults: a systematic review and meta-analysis. J Sports Sci. 2021:39(15):1754-71.

44. European Union. Rare diseases; 2020. Available from: https://ec.europa. eu/info/research-and-innovation/research-area/health-research-andinnovation/rare-diseases_en

45. Aittasalo M, et al. Promoting walking among office employees-evaluation of a randomized controlled intervention with pedometers and e-mail messages. BMC Public Health. 2012;12(1):1-11.

46. Alley $\mathrm{S}$, et al. Web-based video-coaching to assist an automated computer-tailored physical activity intervention for inactive adults: a randomized controlled trial. J Med Internet Res. 2016;18(8):e223.

47. Ashton LM, Morgan PJ, Hutchesson MJ, Rollo ME, Collins CE. Feasibility and preliminary efficacy of the 'HEYMAN' healthy lifestyle program for young men: a pilot randomised controlled trial. Nutr J. 2017;16(1):2. Published 2017 Jan 13. https://doi.org/10.1186/s12937-017-0227-8. 
48. Creel DB, et al. A randomized trial comparing two interventions to increase physical activity among patients undergoing bariatric surgery. Obesity. 2016;24(8):1660-8.

49. Duncan $M$, et al. Effectiveness of a web- and mobile phone-based intervention to promote physical activity and healthy eating in middle-aged males: randomized controlled trial of the ManUp study. J Med Internet Res. 2014;16(6):e136.

50. Fjeldsoe BS, et al. Evaluating the maintenance of lifestyle changes in a randomized controlled trial of the 'get healthy, stay Healthy'program. JMIR mHealth uHealth. 2016;4(2):e42.

51. Golsteijn RHJ, et al. Short-term efficacy of a computer-tailored physical activity intervention for prostate and colorectal cancer patients and survivors: a randomized controlled trial. Int J Behav Nutr Phys Act. 2018;15(1):1-14.

52. Greaney $\mathrm{ML}$, et al. The effect of a weight gain prevention intervention on moderate-vigorous physical activity among black women: the shape program. Int J Behav Nutr Phys Act. 2017;14(1):1-8.

53. Gutiérrez-Martínez $\mathrm{L}$, et al. Effects of a strategy for the promotion of physical activity in students from Bogotá. Rev Saude Publica. 2018;52:79.

54. Hawkins J, et al. Acceptability and feasibility of implementing accelorometry-based activity monitors and a linked web portal in an exercise referral scheme: feasibility randomized controlled trial. J Med Internet Res. 2019;21(3):e12374.

55. Houle J, et al. Innovative program to increase physical activity following an acute coronary syndrome: randomized controlled trial. Patient Educ Couns. 2011;85(3):e237-44.

56. Laing BY, et al. Effectiveness of a smartphone application for weight loss compared with usual care in overweight primary care patients: a randomized, controlled trial. Ann Intern Med. 2014;161(10_Supplement):S5-S12.

57. Müller AM, Khoo S, Morris T. Text messaging for exercise promotion in older adults from an upper-middle-income country: randomized controlled trial. J Med Internet Res. 2016;18(1):e5.

58. Phelan S, et al. Effect of an internet-based program on weight loss for low-income postpartum women: a randomized clinical trial. Jama. 2017;317(23):2381-91.

59. Taylor WC, Paxton RJ, Shegog R, Coan SP, Dubin A, Page TF, et al. Impact of Booster Breaks and Computer Prompts on Physical Activity and Sedentary Behavior Among Desk-Based Workers: A Cluster-Randomized Controlled Trial. Prev Chronic Dis. 2016;13:160231. http://dx.doi.org/10. 5888/pcd13.160231externalicon.

60. Vallance JK, et al. Randomized controlled trial of the effects of print materials and step pedometers on physical activity and quality of life in breast cancer survivors. J Clin Oncol. 2007;25(17):2352-9.

61. Valle CG, Deal AM, Tate DF. Preventing weight gain in African American breast cancer survivors using smart scales and activity trackers: a randomized controlled pilot study. J Cancer Surviv. 2017;11(1):133-48.

62. van der Weegen $S$, et al. It's LiFe! Mobile and web-based monitoring and feedback tool embedded in primary care increases physical activity: a cluster randomized controlled trial. J Med Internet Res. 2015;17(7):e184

63. Watson S, et al. Effect of a web-based behavior change program on weight loss and cardiovascular risk factors in overweight and obese adults at high risk of developing cardiovascular disease: randomized controlled trial. J Med Internet Res. 2015;17(7):e177.

64. R Core Team. R: a language and environment for statistical computing Viennahttps://www.R-project.org: R Foundation for Statistical Computing; 2019.

65. Balduzzi S, Rücker G, Schwarzer G. How to perform a meta-analysis with R: a practical tutorial. Evidence-based Mental Health. 2019;22(4):153-60.

66. Egger $M$, et al. Bias in meta-analysis detected by a simple, graphical test BMJ. 1997;315(7109):629.

67. Sterne JAC, et al. RoB 2: a revised tool for assessing risk of bias in randomised trials. Bmj. 2019;366:14898.

68. Stockwell S, et al. Digital behavior change interventions to promote physical activity and/or reduce sedentary behavior in older adults: a systematic review and meta-analysis. Exp Gerontol. 2019;120:68-87.
69. Davies CA, et al. Meta-analysis of internet-delivered interventions to increase physical activity levels. Int J Behav Nutr Phys Act. 2012;9(1):1-13.

70. Laranjo $L$, et al. Do smartphone applications and activity trackers increase physical activity in adults? Systematic review, meta-analysis and metaregression. Br J Sports Med. 2020:bjsports-2020-102892.

71. Conroy DE, Yang C-H, Maher JP. Behavior change techniques in top-ranked Mobile apps for physical activity. Am J Prev Med. 2014;46(6):649-52.

72. Yang $\mathrm{C}-\mathrm{H}$, Maher JP, Conroy DE. Implementation of behavior change techniques in Mobile applications for physical activity. Am J Prev Med. 2015;48(4):452-5.

73. Bondaronek P, et al. Quality of publicly available physical activity apps: review and content analysis. JMIR mHealth uHealth. 2018;6(3):e53.

74. Samdal GB, et al. Effective behaviour change techniques for physical activity and healthy eating in overweight and obese adults; systematic review and meta-regression analyses. Int J Behav Nutr Phys Act. 2017;14(1):42.

75. Schoeppe $\mathrm{S}$, et al. Apps to improve diet, physical activity and sedentary behaviour in children and adolescents: a review of quality, features and behaviour change techniques. Int J Behav Nutr Phys Act. 2017;14(1):83.

76. Yardley L, et al. Understanding and promoting effective engagement with digital behavior change interventions. Am J Prev Med. 2016;51(5):833-42.

77. Miller S, et al. A framework for analyzing and measuring usage and engagement data (AMUsED) in digital interventions. J Med Internet Res. 2019;21(2):e10966.

78. Yoon $\mathrm{H}$, et al. Older adults' internet use for health information: digital divide by race/ethnicity and socioeconomic status. J Appl Gerontol. 2018;39(1):105-10.

79. Mitsutake $\mathrm{S}$, et al. Associations of eHealth literacy with health behavior among adult internet users. J Med Internet Res. 2016;18(7):e192.

80. Britt RK, et al. eHealth literacy and health behaviors affecting modern college students: a pilot study of issues identified by the American college health association. J Med Internet Res. 2017;19(12):e392.

81. Schüz B, et al. Socioeconomic status as a moderator between social cognitions and physical activity: systematic review and meta-analysis based on the theory of planned behavior. Psychol Sport Exerc. 2017;30:186-95.

82. Michie S, van Stralen MM, West R. The behaviour change wheel: a new method for characterising and designing behaviour change interventions. Implement Sci. 2011;6(1):42

83. Pampel FC, Krueger PM, Denney JT. Socioeconomic disparities in health behaviors. Annu Rev Sociol. 2010;36:349-70.

84. Lustria MLA, et al. A meta-analysis of web-delivered tailored health behavior change interventions. J Health Commun. 2013;18(9):1039-69.

85. Ryan K, Dockray S, Linehan C. A systematic review of tailored eHealth interventions for weight loss. Digital Health. 2019;5:2055207619826685.

86. Crawford A, Serhal E. Digital health equity and COVID-19: the innovation curve cannot reinforce the social gradient of health. J Med Internet Res. 2020;22(6):e19361.

87. Yardley $L$, et al. The person-based approach to enhancing the acceptability and feasibility of interventions. Pilot Feasib Stud. 2015;1(1):1-7.

88. Yardley $L$, et al. The person-based approach to intervention development: application to digital health-related behavior change interventions. J Med Internet Res. 2015;17(1):e30.

89. King AC, et al. Leveraging citizen science and information Technology for Population Physical Activity Promotion. Transl J Am College Sports Med. 2016;1(4):30-44.

\section{Publisher's Note}

Springer Nature remains neutral with regard to jurisdictional claims in published maps and institutional affiliations. 